DOI: $10.1515 /$ plass-2016-0016

Abdul Razzaq ${ }^{1}$, Madiha Rashid ${ }^{1}$, Talal Ahmad Shfique ${ }^{1}$, Abdul Hafeez $^{1}$, Hafiz Muhammad Jhanzab ${ }^{1}$, Ghulam Shabir ${ }^{2}$, Zahid Hussain Shah ${ }^{3 *}$

${ }^{1}$ Crop Physiology Lab, Department of Agronomy, Pir Mehr Ali Shah Arid Agriculture University Rawalpindi, Pakistan. PC $-46300 ;{ }^{2}$ Department of Plant Breeding and Genetics, Pir Mehr Ali

Shah Arid Agriculture University Rawalpindi, Pakistan. PC - 46300; ${ }^{3}$ Department of Arid Land Agriculture, King Abdul Aziz University Jeddah, Saudi Arabia.

PC -21577;* Corresp. Author's e-mail: shahzahid578@hotmail.com

\title{
ABA SENSITIVITY AS A CRITERION FOR DROUGHT TOLERANCE IN WHEAT (TRITICUM AESTIVUM L.) CULTIVARS
}

\section{ABSTRACT}

Criteria used for evaluating drought tolerance of wheat cultivars demanding more time and efforts are usually not efficient and conclusive. Present study was conducted to evaluate ABA sensitivity as a criterion for evaluating drought tolerance of wheat cultivars at an early stage. Ten cultivars of wheat were subjected to drought at 3-leaf stage to select the most sensitive and two tolerant cultivars using mortality rate. Tatara was found the most susceptible whereas GA-2002 and Chakwal-50 were the cultivars with maximum drought tolerance. These cultivars were used to study sensitivity to applied absicic acid (based on germination index and relative growth inhibition rate) and physiological responses (leaf water content, chlorophyll stability index, coefficient of relative inhibition and proline accumulation) under drought stress. Highest ABAsensitivity was recorded in GA-2002 and Chakwal-50 whereas Tatara manifested minimum sensitivity. ABAsensitivity corresponded to physiological indices of drought tolerance. Results show that ABA-sensitivity is an efficient criterion that can be used to evaluate drought tolerance of wheat cultivars at early stage.

Keyword: ABA, chlorophyll, inhibition, proline

Abbreviations: ABA (absicic acid), RGIR (relative growth inhibition rate), GI (germination index), CSI chlorophyll stability index), CRI (coefficient of relative inhibition)

\section{INTRODUCTION}

Drought is a worldwide problem significantly affecting overall crop production (Dhanda et al. 2004). Current global weather modification has made this condition more serious. In arid and semi-arid areas drought stress is a severe problem that is critically affecting crop production and causing huge loss in cropping globally. Major proportion of wheat, the most im-

Communicated by Grzegorz Żurek 
portant staple food world over, is grown under arid and semiarid areas constituting about $60 \%$ of global land with large fluctuations in the amount and frequency of rainfall. Insufficient water is the primary limitation to wheat production in these areas that influences all aspects of biology (Bayoumi et al. 2008) declining average yield of the crops. In general, drought is one of the most important constrains of biomass production and evolving/selecting drought resistance wheat cultivars seems the most plausible solution for drought prone areas. Development of drought tolerant cultivars increases crop production and yield stability under water deficit stress (Siddique et al. 2000). To augment wheat production in future development of new tolerant cultivars is crucial. Drought has adverse effect on the growth and yield of crops. Physiological response to drought arises from the changes in cellular gene expression.

Plants are evaluated for drought tolerance based on their physiological responses. Leaf water content is commonly used as an indicator for both susceptibility and resistance to water stress in crops (Sanchez-Rodriguez et al. 2010). Decrease in relative water content of leaves leads to stomatal closure which in turn reduces photosynthesis rate (Cornic, 2000). Reduction in chlorophyll synthesis under drought may lead to decline in photosynthesis. High chlorophyll stability index specifies plant's tolerance under water deficit (Ramesh and Devasenapathy, 2006).

Regulation of cell solutes by lowering down osmotic potential is believed to be due to the osmotic regulations (Turner and Jones, 1980). Accumulation of cytocompatible osmolytes is an important adjustment to mitigate the effect of drought. Various organic and inorganic solutes are accumulated to maintain the driving gradient for water uptake by maintaining osmotic and turgor pressure. Solute accumulation such as proline, betaine and polyols facilitate osmotic adjustment (Shao et al. 2005). Proline is the most extensively studied organic solute and its role in conferring osmotolerance has been widely reported (Bajji et al. 2000). High proline accumulation is a distinctive feature of most plants under various environmental stresses (Kavi et al. 2005) and has been used as a criterion for assessing drought resistance of wheat varieties (Gunes et al., 2008). Wheat plants under drought stress show increase in proline content (Tatar and Gevrek, 2008).

Complexity of drought traits with low genetic variance of yield component under stress condition limits conventional breeding making the screening procedure difficult. Drought resistance being a multiple gene-control quantitative trait and wheat genome being larger, less progress has been made in improving drought resistance of wheat. Identification and selection of secondary traits contributing to drought resistance and yield stability in water limited environments may be used for screening of drought tolerant wheat cultivars. Sensitivity to $\mathrm{ABA}$ can be used successfully for selection of drought tolerant wheat cultivars in short duration of time.

Absicic acid (ABA) sensitivity has been found associated with drought tolerance (Cutler et al. 1996) and increased cold tolerance in wheat (Kobayashi et al. 2008). ABA hypersensitive mutants are expected to close stomata at lower concentrations of ABA resulting in decreased transpiration 
under drought stress (Wang et al. 2005). Exogenous application of ABA prevents embryonic germination. Inhibitory effects of ABA are less in genotypes susceptible to drought than the resistant ones (Thomas et al. 1991). Consequently ABA sensitivity can play a vital role in selecting drought tolerant cultivars (Zhang et al, 2006). ABA sensitivity can be used to improve drought tolerance of wheat without affecting yield (Schramm et al. 2008). Visualizing the potential importance of ABA sensitivity for screening drought tolerant cultivars present study was conducted to compare ABA sensitivity with other drought tolerant traits to establish its efficacy for selecting drought tolerant wheat cultivars.

\section{MATERIALS AND METHODS}

\section{Evaluating the most susceptible and drought tolerant cultivars}

First step of the study was to select the two most drought tolerant and one the most sensitive cultivar among the ten high yielding wheat cultivars including Farid-06, Faisalabad-08, AARI-10, Sehar-06, GA-2002, Tatara, Wafaq-01, Meraj-2008, Chakwal-50, NARC-09. Seeds were sown in pots for germination and seedling growth. At three leaf stage application of water was withheld to impose drought. To categorize cultivars as drought tolerant or susceptible data on mortality rate was recorded on 3, 4, 5 and 6 days after withholding water. The experiment was laid out using completely randomized design (CRD) with four replications. Mortality rate was calculated by following equation:

$$
M R=\frac{N o_{D S}}{N o_{T S} \times N o_{D}} \times 100
$$

where $M R$-mortality rate, $N o_{D S}$-number of dead seedlings, $N o_{T S}-$ number of total seedlings and $\mathrm{No}_{D}$ - number of days

The cultivar with highest mortality rate was regarded the most susceptible whereas two with lowest mortality rate were selected as drought tolerant cultivars. The most susceptible one and two tolerant cultivars determined in this step were used for further experimentation.

\section{Abscisic Acid (ABA) Sensitivity}

A separate experiment was conducted to measure ABA sensitivity of selected cultivars. Two pieces of filter papers were placed into each labeled Petri dish. Water or appropriate ABA solution $(10,20,25$ and $30 \mu \mathrm{M})$ was added in equal amounts to thoroughly soak the filter paper. In each Petri dish, 20 seeds of each selected (the most sensitive and drought tolerant) cultivars were placed on the surface of the filter paper with the crease of each seed facing down. The Petri dishes were incubated at $20^{\circ} \mathrm{C}$ and about $95 \%$ relative humidity in the dark. CRD with four replications was used for layout of the experiment. Each dish was examined daily to record germinated seeds. 
Weighted germination index (WGI) was calculated by following formula (Reddy et al., 1985):

$$
W G I=\frac{\left(8 \times n_{1}+7 \times n_{2}+\cdots+1 \times n_{8}\right)}{8} \times N
$$

where;

$N$ - the total seeds placed in the plate, $n_{l}$ - the number seeds that germinated on the $1^{\text {st }}$ day receiving the maximum weight of 8 for the number of days the germination was recorded. Less weight $7 \ldots 1$ agreed to the number of seeds germinated $n_{2} \ldots n_{8}$ on each subsequent day.

ABA sensitivity was calculated from $W G I$ by following formula:

$$
A B A_{\text {sensitivity }}=1-W G I
$$

\section{Relative Growth Inhibition Rate}

Relative growth inhibition rate (RGIR) also gives an estimation of ABA sensitivity Therefore, RGIR was also estimated in the study. Seeds were germinated in petri dishes fitted with filter papers soaked with distilled water and 10,20,25 and $30 \mu \mathrm{M}$ ABA. CRD with four replications was employed for experime4ntal layout. Seedlings were allowed to grow till 2-3 leaf stage. Then, average fresh weight of seedlings was measured from each petridish.

RGIR was calculated by the following formula ( Kurahashi et al. 2009):

$$
R G I R=\left(1-\frac{A F W_{+A B A}}{A F W_{-A B A}}\right) \times 100 \%
$$

where;

$A F W_{+A B A}$ - average fresh weight with $\mathrm{ABA}$ and $A F W_{-A B A}$ - average fresh weight without ABA.

Another experiment was conducted for physiological evaluation of drought tolerance/susceptibility of the selected cultivars (the most susceptible one and two drought tolerant). Seeds of three cultivars were sown in pots. After germination seedlings were allowed to grow up to three leaf stage under normal condition. Then water application was withheld exposing the plants to water deficit stress. The experimental layout was CRD with four replications. Data on following parameters was recorded:

\section{Leaf water content}

On $4^{\text {th }}$ day of stress leaf water content was measured. Two leaves were cut from each replication and weighed immediately for fresh weight (FW). Then leaves were placed in oven at $70^{\circ} \mathrm{C}$ for 36 hours for dry weight (DW). Water content (WC) was calculated as: 


$$
W C=\frac{(F W-D W)}{D W} \times 100 \%
$$

where;

$W C(\%)$ - water content, $F W$ - fresh weight and $D W$ - dry weight

\section{Chlorophyll Stability Index}

Chlorophyll meter was used to measure total chlorophyll content of plants in each replicate on $4^{\text {th }}$ day of stress. Chlorophyll stability index (CSI) was calculated by following formula:

$$
\operatorname{CSI}=\frac{\operatorname{ChCSP}}{\operatorname{ChCCP}} \times 100 \%
$$

where;

CSI (\%) - chlorophyll stability index, ChCSP - chlorophyll content of stressed plants and $C h C C P$ - chlorophyll content of control plants.

\section{Coefficient of Relative Inhibition (CRI)}

Biomass of stressed and unstressed plants was recorded at $3^{\text {rd }}, 5^{\text {th }}$ and $7^{\text {th }}$ day of water stress and CRI was calculated by using formula given below:

$$
C R I=\frac{B u n S P-B S P}{B u n S P} \times 100 \%
$$

where;

$C R I(\%)$ - coefficient of relative inhibition, BunSP - biomass of unstressed plant and $B S P$ - biomass of stressed plants.

\section{Proline content}

To determine proline content leaf tissues were rinsed twice with distilled water and oven dried at $75^{\circ} \mathrm{c}$ for 3 days. Each dried leaf sample was crushed in mortar with a pestle. Sulfosalicyclic acid solution $(10 \mathrm{ml})$ was added to each tube containing $0.5 \mathrm{~g}$ of the leaf. After two days, $1 \mathrm{ml}$ of the extract was reacted with $1 \mathrm{ml}$ of glacial acetic acid and $1 \mathrm{ml}$ ninhydrin $(1.25 \mathrm{~g}$ ninhydrin warmed in $30 \mathrm{ml}$ glacial acetic acid and $20 \mathrm{ml}, 6 \mathrm{M}$ phosphoric acid until resolved) in a water bath $\left(100^{\circ} \mathrm{C}\right)$ for an hour. The reaction was terminated in an ice bath to stabilize the purple color of the extract. Then, $0.2 \mathrm{ml}$ toluene was added to each tube and the absorbance of top purple aqueous layer was measured at $520 \mathrm{~nm}$ in a spectrophotometer.

The data collected for all parameters was subjected to analysis of variance technique using Statistix 8.1 software to determine significance of the treatments. Means of significant effects were separated using the LSD at 5 percent probability level. ABA sensitivity and ABA induced growth inhibition were correlated with drought parameters to establish ABA sensitivity as a criterion for assessing drought tolerance of wheat cultivars. 


\section{RESULTS AND DISCUSSION}

\section{Mortality Rate}

Mortality rate is the number of dead plants out of total plants expressed in percentage. Mortality rate of different wheat cultivars at 2 - 3 leaf stage is depicted in Fig-1. An increase in mortality rate was observed with increase in stress duration from 3 to 6 days in all cultivars under study. However, mortality rate differed significantly among wheat cultivars at $5 \%$ significance level. Lowest mortality rate was recorded in GA-2002 (28.9 \%) followed by Chakwal-50 (38.2\%). Whereas highest mortality rate was observed in Tatara (92.6 \%) followed by Faisalabad-08 (87.8\%). Other cultivars (Farid-06, Faisalabad-08, AARI-10, Sehar-06, GA-2002, Tatara, Wafaq-01, Meraj-2008, Chakwal-50, NARC-09) also had significant higher mortality rate than G.A-2002 and Chakwal-50. Wafaq-01 and Meraj-2008 showed non significant difference.

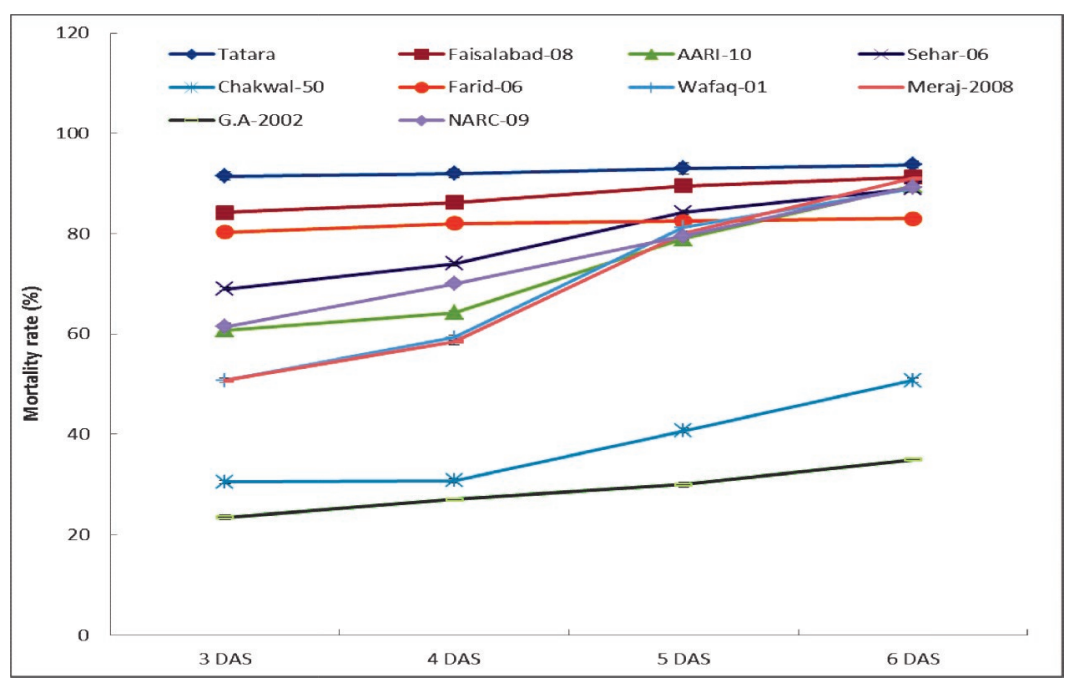

Fig-1: Mortality rate of wheat cultivars at $3^{\text {rd }}, 4^{\text {th }}, 5^{\text {th }}$, and $6^{\text {th }}$ day after stress

Chakwal-50 and GA-2002 are the best wheat cultivars that gave good germination and coleoptile length under stress conditions (Qayyum et al. 2011). Balla et al. 2006 found differences between genotypes regarding their tolerance to environmental stress, allowing selection to be made for better tolerance. Propagation of drought tolerant cultivars may ensure safety of wheat production.

Based on mortality rate Tatara was classified as the most drought sensitive cultivar and GA-2002 followed by Chakwal-50 as the cultivars with highest tolerance. These three cultivars viz. GA-2002, Chakwal-50 and Ta- 
tara were then used for further experimentation to establish ABA sensitivity as a criterion for drought tolerance of wheat cultivars.

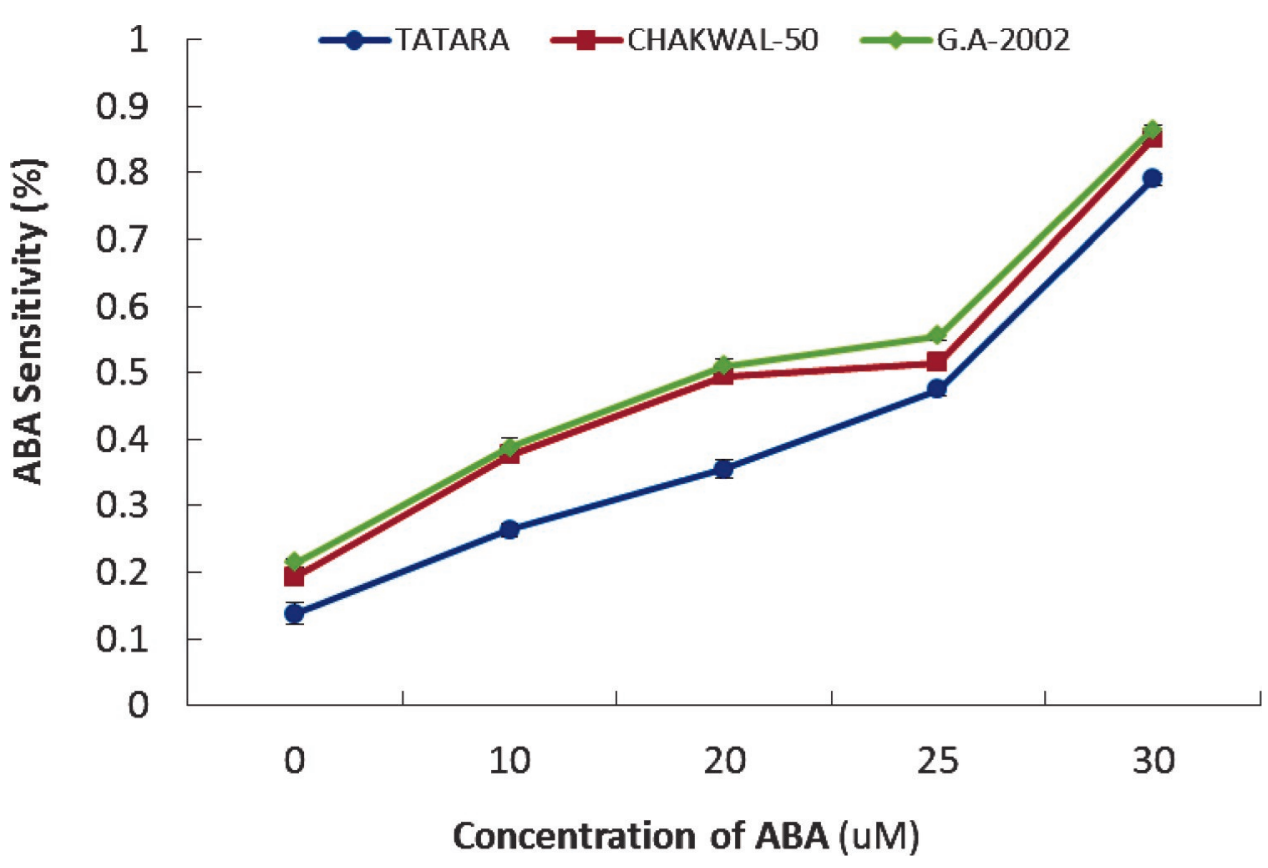

Fig. 2. Abscisic acid sensitivity (\%) of three wheat cultivars in response to different levels of ABA

ABA sensitivity estimated by weighted germination index (WGI) ABA sensitivity differed significantly in wheat cultivars at $5 \%$ significance level (Fig-2). GA-2002 exhibited maximum ABA sensitivity $(0.506 \%)$ followed by Chakwal$50(0.486 \%)$ and minimum was observed in Tatara $(0.402 \%)$. ABA sensitivity corresponds to drought tolerance of the cultivars. The most sensitive cultivar i.e. Tatara manifested minimum sensitivity whereas maximum sensitivity was observed for GA-2002, the most drought tolerant cultivar followed by Chakwal50, the next drought tolerant cultivar. Exposure of wheat cultivars from 10 to 20 $\mu \mathrm{M}$, ABA widened the gap between sensitivity of Tatara, GA-2002 and Chakwal-50 compared with control. However, further increase in the level of ABA to 25 and $30 \mu \mathrm{M}$ minimized the difference in sensitivities of the cultivars. Difference in the sensitivity of GA-2002 and Chakwal-50 became nonsignificant at $30 \mu \mathrm{M}$ but still the sensitivity of these two cultivars was significantly higher than that of Tatara, the drought sensitive cultivar.

\section{ABA Sensitivity}

RGIR and WGI were used to assess ABA sensitivity of selected wheat cultivars. Significant differences were observed in RGIR of the three cultivars at 5\% probability level Fig-3. Maximum RGIR was recorded in GA-2002 (50.6) followed by Chakwal-50 (45.8) whereas minimum RGIR (34.4) was observed in Tatara. Relative growth inhibition rate (RGIR) in response to applied ABA cor- 
responds to drought tolerance of the selected cultivars. Drought tolerant cultivars viz. GA-2002 and Chakwal-50 manifested relatively higher RGIR compared with drought sensitive cultivar i.e. Tatara. Maximum difference in RGIR of drought tolerant and sensitive cultivars was recorded at 10 and $20 \mu \mathrm{M}$, ABA. Further increase in concentration of ABA lessened the difference in RGIR of the cultivars. Raikhel et al. (1986) observed stunted growth of young wheat plants by exogenous $\mathrm{ABA}(10 \mu \mathrm{M})$. While seedlings grown on $1 \mu \mathrm{M}$ ABA were nearly normal in size and as the concentrations of ABA increased seedlings growth was reduced. Our results support the hypothesis that drought tolerant cultivars show higher sensitivity to exogenous ABA whereas drought susceptible cultivars show low sensitivity.

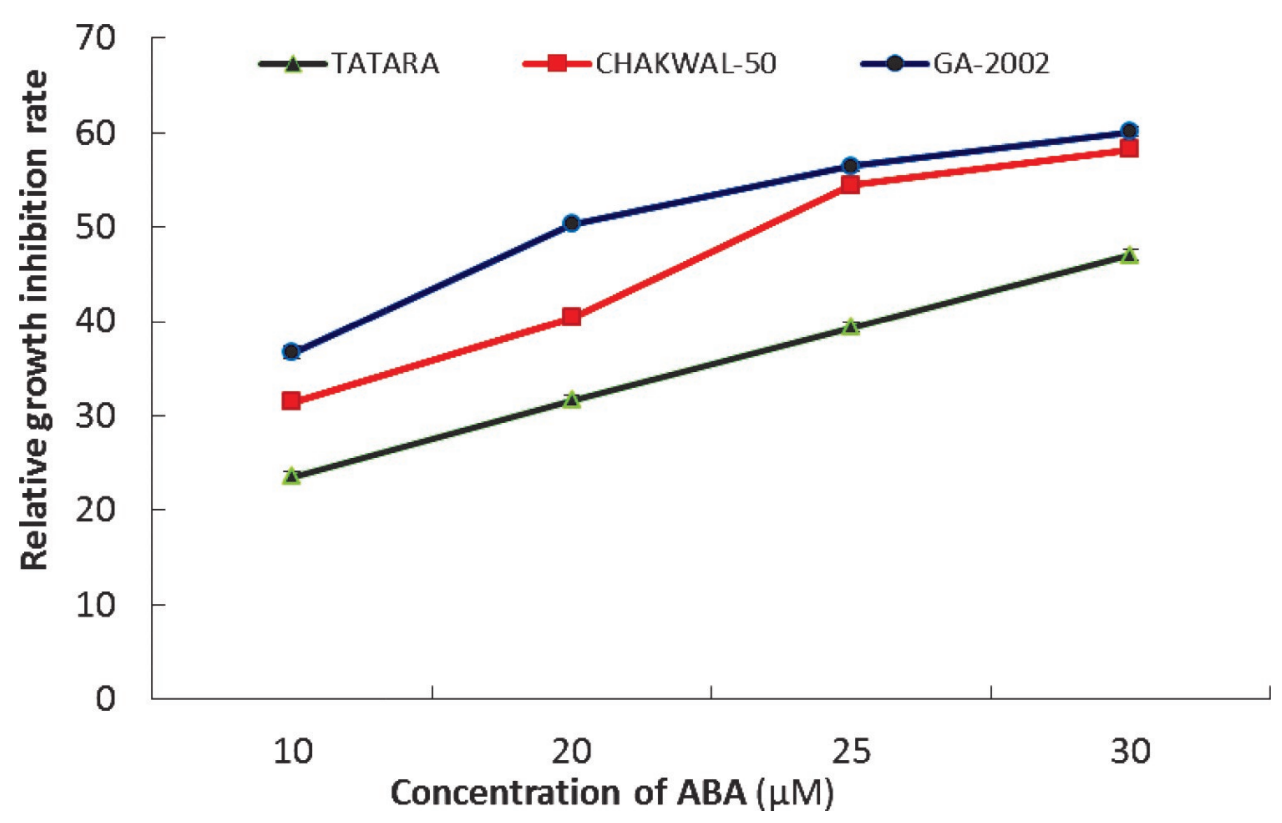

Fig. 3. Relative growth inhibition rate of three wheat cultivras in response to ABA

Exogenous ABA inhibits germination in wheat grains (Williamson and Quatrano, 1988) and can be used to measure ABA sensitivity. Our results show higher ABA sensitivity based on WGI and RGIR in drought tolerant cultivars compared with drought sensitive ones. Thus, ABA can play vital role in selecting for drought tolerant cultivars (Zhang et al. 2006). Susceptible cultivars have relatively low sensitivity to $\mathrm{ABA}$ as compared to tolerant cultivars (Nyachiro et al. 2002). Drought susceptible genotypes are less sensitivity to ABA (Thomas et al. 1991). Scramm et al. (2008) observed that ABA hypersensitive mutants of wheat appeared more green and turgid with low transpiration. A positive relationship between stomatal oscillation, closure and sensitivity to ABA was reported in Arabidopsis (Zhang et al. 2009). In our study drought tolerance was found related with ABA sensitivity. Tatara, the most susceptible cultivar exhibited lowest sensitivity to ABA while highest ABA sensitivity was measured in 
drought tolerant cultivars viz. GA-2002 and Chakwal-50. ABA sensitivity is closely related to drought stress tolerance (Kurahashi et al. 2009) and may be used as a reliable and efficient criterion for selecting drought tolerant wheat cultivars at early stage in short period of time. Role of ABA in establishing drought tolerance has been reported in Arabidopsis, maize, tobacco, wheat and tomato (Finkelstein and Gibson, 2002).

\section{Physiological responses to drought}

Leaf water content: Physiological responses to drought such as water content, chlorophyll stability index (CSI), proline accumulation and coefficient of relative inhibition (CRI) provide a good estimation of drought tolerance of plants. Water content of wheat cultivars under water stress at 2-3 leaf stage differed significantly in wheat cultivars at 5\% significance level (Table-1). Maximum water content was noticed in GA-2002 (429\%) followed by Chakwal-50 (413.3 $\%)$. Tatara (384 \%) exhibited the minimum water content under stress. Leaf water content of three cultivars matched with their level of drought tolerance. Decline in water content was recorded from drought tolerant cultivars to susceptible one. Water contents of the cultivars were consistent with their tolerance level. Highest water content was recorded in the most drought tolerant cultivar (G.A-2002) followed by (Chakwal-50) and lowest in the most sensitive one (Tatara). Tolerant cultivars maintain of relatively higher water content under water deficit condition (Khanna-Chopra and Selote, 2007). Leaf water content is a parameter to judge the drought tolerant capability and photosynthesis of plants. It represents turgidity and ability of plant to maintain water uptake under drought stress on which physiological functioning of plants depends. Almeselmani (2011) demonstrated that for improvement of drought tolerance in wheat, Chlorophyll content and relative water content were the indices of drought tolerance. Guoth et al. (2009a) recorded considerable decline of water content was in the sensitive cultivar compared with drought tolerant ones. Water content, an index of drought tolerance (Almeselmani, 2011), can be used as a physiological parameter to evaluate the capacity of drought tolerance and photosynthetic efficiency of wheat. Resistant cultivars maintain better leaf water relations as compared to drought sensitive ones (Khanna-Chopra and Selote, 2007).

Chlorophyll stability index: Chlorophyll stability index (CSI) of tolerant and susceptible cultivars differed significantly at $5 \%$ significance level (Table-1). Maximum CSI was noticed in GA-2002 and Chakwal-50 (68.6\% and 66.5\%), respectively and minimum value was observed in Tatara $(39.1 \%)$. CSI corresponds to the tolerance level of wheat cultivars under study. Highest CSI was recorded in GA-2002, the most drought tolerant cultivar followed by Chakwal50 and lowest in drought tolerant susceptible i.e. Tatara. Chlorophyll content is an index of drought tolerance of wheat (Almeselmani, 2011) Higher CSI specifies plant's tolerance under water deficit (Ramesh and Devasenapathy, 2006). In our experiments GA-2002 is the most tolerant and Tatara the most sensitive cultivar. Continuous water stress inhibits chlorophyll synthesis in wheat cultivars. 
Significant decrease in chlorophyll content was recorded in susceptible compared with drought tolerant wheat cultivar (Tas and Birol, 2007).

Physiological responses of wheat cultivars to drought stress

Table 1

\begin{tabular}{|c|c|c|c|c|c|c|c|c|c|c|}
\hline \multirow{2}{*}{$\begin{array}{l}\text { Wheat } \\
\text { cultivar }\end{array}$} & \multirow{2}{*}{$\begin{array}{c}\text { Water } \\
\text { Content } \\
{[\%]}\end{array}$} & \multirow{2}{*}{ CSI } & \multicolumn{4}{|c|}{$\begin{array}{l}\text { Proline content } \\
{\left[\mu \mathrm{g} \times \mathrm{g}^{-1} \text { of leaf }\right]}\end{array}$} & \multicolumn{4}{|c|}{ Coefficient of relative inhibition } \\
\hline & & & $3 \mathrm{DAS}$ & 5 DAS & 7 DAS & Means & 3 DAS & 5 DAS & 7 DAS & Means \\
\hline GA.2002 & $429 * A^{* *}$ & $68.6 \mathrm{~A}$ & $0.835 \mathrm{ef}$ & $1.317 \mathrm{~d}$ & $2.00 \mathrm{ab}$ & $1.384 \mathrm{ab}$ & $0.128 \mathrm{~d}$ & $0.259 \mathrm{c}$ & $0.402 b$ & $0.263 b$ \\
\hline Chakwal-50 & $413.3 \mathrm{~B}$ & $66.5 \mathrm{~A}$ & $0.854 \mathrm{f}$ & $1.509 \mathrm{~cd}$ & $2.17 \mathrm{a}$ & $1.511 \mathrm{a}$ & $0.144 d$ & $0.296 \mathrm{c}$ & $0.396 b$ & $0.278 b$ \\
\hline \multirow[t]{2}{*}{ Tatara } & $384.9 \mathrm{C}$ & $39.1 \mathrm{~B}$ & $0.81 \mathrm{f}$ & $1.175 \mathrm{de}$ & $1.77 \mathrm{bc}$ & $1.251 \mathrm{~b}$ & $0.0407 \mathrm{e}$ & $0.417 \mathrm{~b}$ & $0.639 \mathrm{a}$ & $0.365 a$ \\
\hline & & & $0.833 c$ & $1.333 b$ & $1.979 \mathrm{a}$ & & $0.104 c$ & $0.324 b$ & $0.479 \mathrm{a}$ & \\
\hline
\end{tabular}

*Each value is the mean of four separate observations,

**Means not sharing a letter in common differ significantly at $\mathrm{P}>0.05$

Coefficient of Relative Inhibition: Coefficient of relative inhibition (CRI) is a measure of growth inhibition. CRI of drought sensitive and tolerant wheat cultivars differed significantly (Table-1). Statistically similar growth inhibition was observed in tolerant cultivars (GA-2002 and Chakwal-50) throughout the stress duration. Nonetheless, significantly higher CRI was manifested by Tatara, the drought sensitive cultivar. Increased exposure time was accompanied by an increase in CRI. Stress induced growth inhibition may be ascribed to decreased water content and degradation of chlorophyll.

Proline accumulation: Proline accumulation of in selected cultivars of wheat differed significantly in response to drought (Table-1). Increase in stress duration was accompanied by increase in proline accumulation in all the three cultivars. On average, Chakwal-50 accumulated significantly more proline $\left(1.5107 \mu \mathrm{g} \times \mathrm{g}^{-1}\right)$ than Tatara $\left(1.2510 \mu \mathrm{g} \times \mathrm{g}^{-1}\right)$ and GA-2002 $\left(1.3843 \mu \mathrm{g} \times \mathrm{g}^{-1}\right)$. Difference in proline accumulation may not be regarded decisive in differentiating tolerance level of the cultivars.

Proline plays a role in osmotic adjustment by plants (Delauney and Verma, 1993). It has a complex effect suggesting its importance in stress tolerance. Proline accumulation under stress helps the plants to maintain cell turgor (Valentovic et al. 2006), stabilizes cellular proteins/membranes (Errabii et al. 2006) and scavenges free radicals (Kavi et al. 2005). Increased proline accumulation in wheat under stress conditions (Tatar and Gevrek, 2008) helps plants evade harmful effects of drought (Vendruscolo et al. 2007)

\section{CONCLUSION}

Results of the study explicitly reveal that ABA sensitivity may be used as a reliable, efficient and appropriate criterion for screening drought tolerant wheat cultivars in short duration. The cultivars with higher sensitivity to drought had high leaf water content, more CSI, accumulated more proline and low CRI. Hence, ABA sensitivity can be used positively for differentiating wheat cultivars for their drought tolerance. The best level of exogenous ABA is 
10 to $20 \mu \mathrm{M}$ for this purpose. At higher concentrations of ABA differences in sensitivity lessens making it difficult to distinguish sensitive cultivars from drought tolerant ones.

\section{REFERENCES}

Almeselmani, M. 2011. Effect of Drought on Different Physiological Characters and Yield Component in Different Varieties of Syrian Durum Wheat. J. Agric. Sci. 3 (3)

Bajji, M., S. Lutts, and J. M. Kinet. 2000. Physiological changes after exposure to and recovery from polyethylene glycol-induced water deficit in callus cultures issued from durum wheat (Triticum durum Desf.) cultivars differing in drought resistance. J. Plant Physiol. 156 (1): 75-83

Balla, K., Z. Bed and O. Veisz. 2006. Effect of heat and Drought stress on the photosynthetic processes of wheat. Cereal Research Communications. 34 (1): 381-385

Bayoumi, T. Y., M. H. Eid and E. M. Metwali. 2008. Application of physiological and biochemical indices as a screening technique for drought tolerance in wheat genotypes. Afr. J. Biotechnol. 7: 2341-2352.

Cornic, G. 2000. Drought stress inhibits photosynthesis by decreasing stomatal aperture not by affecting ATP synthesis. Trends Plant Sci. 5: 187-198.

Cutler, S., M. Ghassemian, D. Bonetta, S. Cooney and P. McCourt. 1996. Science 273: 1239-41.

Delauney, A. J. and D. P. S. Verma. 1993. Proline biosynthesis and osmoregulation in plants. Plant J. 4: 215 223.

Dhanda, S.S., G.S. Sethi and R. K. Behl. 2004. Indices of drought tolerance in wheat genotypes at early stages of plant growth. J. Agron. Crop Sci. 190 (1): 6-12.

Errabii, T., C.B. Gandonou, H. Essalmani, J. Abrini, M. Idomar and N.S. Senhaji. 2006. Proline and ion accumulation in sugarcane callus cultures under drought-induced osmotic stress and its subsequent relief. Afri. J. Biotechnol. 5: 1148-1493.

Finkelstein, R., S.I. Gibson. 2002. ABA and sugar interactions regulating development: "cross-talk" or "voices in a crowd". Curr. Opin. Plant Biol. 5:26-32.

Guoth, A., I. Tari, A. Galle, J. Csiszar, A. Pecsvaradi, L. Cseuz and L. Erdei. 2009. Comparison of the drought stress responses of tolerant and sensitive wheat cultivars during grain filling: changes in flag leaf photosynthetic activity, ABA levels and grain yield. J. Plant Growth Reg. 28: 167-176.

Gunes A., D. Pilbeam, A. Inal. and S. Coban. 2008. Influence of silicon on sunflower cultivars under drought stress, I: Growth, antioxidant mechanisms and lipid peroxidation. Commun. Soil Sci. Pl. Nutr. 39: 18851903.

Kavi K., S. Sangam, R.N. Amrutha, P.S. Laxmi, K.R. Naidu, K.R.S.S. Rao, S. Rao, K.J. Reddy, P. Theriappan and N. Sreenivasulu. 2005. Regulation of proline biosynthesis, degradation, uptake and transport in higher plants: its implications in plant growth and abiotic stress tolerance. Curr. Sci. 88: 424-438.

Khanna-Chopra, R. and D. S. Selote. 2007. Acclimation to drought stress generates oxidative stress tolerance in drought resistant than susceptible wheat cultivar under field conditions. Environ. Exp. Bot. 60: 276283.

Kobayashi, F., S. Takumi and C. Nakamura. 2008. Increased freezing tolerance in an ABA-hypersensitive mutant of common wheat. J. Plant Physiol. 165: 224-32.

Kurahashi, Y., A. Terashima and S. Takumi. 2009. Variation in Dehydration Tolerance, ABA Sensitivity and Related Gene Expression Patterns in D-Genome Progenitor and Synthetic Hexaploid Wheat Lines. Int. J. Mol. Sci., 10: 2733-2751.

Nyachiro, J.M., F. R. Clarke, R. M. DePauw, R. E. Knox and K. C. Armstrong. 2002. The effects of cis-trans ABA on embryo germination and seed dormancy in wheat. Euphytica., 126: 129-133.

Qayyum, A., A. Razzaq, M. Ahmad and M.A. Jenks. 2011. Water stress causes differential effects on germination indices, total soluble sugar and proline content in wheat (Triticum aestivum L.) genotypes. African. J. Biotech., 10 (64): 14038-14045.

Raikhel, N.V., B.A. Palevitz and C.H. Haigler. (1986). Absicic acid control of lectin accumulation in wheat seedling and callus culture: effect of exogenous ABA and flouridone. Plant Physiol. 80: 167-171.

Ramesh, H., and P. Devasenapathy. 2006. Physiological Response of Cowpea in a Rainfed Alfisol Ecosystem to the Impulse of Soil Moisture Conservation Practices. Gen. Appl. Plant Physiol. 32 (3-4): 181-190.

Reddy, L.V., R. J. Metzger and T. M. Ching. 1985. Effect of temperature on seed dormancy of wheat. Crop Sci. 25: 455-458

Sanchez-Rodriguez, E., M. Rubio-Wilhelmi, L. M. Cervilla, B. Blasco, J. J. Rios, M. A. Rosales, L. Romero and J. M. Ruiz. 2010. Genotypic differences in some physiological parameters symptomatic for oxidative stress under moderate drought in tomato plants. Plant Sci. 178: 30-40.

Schramm E., J. Abellera, L. Strader, K.G. Campbell and C.M. Steber. 2008. Can ABA signaling be used to develop drought tolerant wheat. The 11th International Wheat Genetics Symposium proceedings, Ed, Rudi Appels Russell Eastwood Evans Lagudah Peter Langridge Michael Mackay Lynne. Washington State University. 
Shao, L., L.T. Young and J.F. Wang. 2005. Chronic treatment with mood stabilizers lithium and valproate prevents excitotoxicity by inhibiting oxidative stress in rat cerebral cortical cells. Biol. Psychiatry. 58: 879-84.

Siddique, M.R.B., A. Hamid and M.S. Islam. 2000. Drought stress effects on water relation of wheat. Bot. Bull. Acad. Sin., 41 (1): 35-39.

Tas, S., and T. Birol. 2007. Some Physiological Responses of Drought Stress in Wheat Genotypes with Different Ploidity in Turkiye. World J. Agric. Sci. 3 (2): 178-183.

Tatar, O. and M. N. Gevrek. 2008. Influence of water stress on proline accumulation, lipid peroxidation and water content of wheat. Asian J. Plant Sci. 7(4): 409-412.

Thomas, T. L., J. Vivekananda and M. A. Bogue. 1991. ABA regulation of gene expression in embryos and mature plants. In: W.J. Davies \& H.G. Jones (Eds.), Abscisic Acid Physiology and Biochemistry, Environmental Plant Biology Series, Bios Scientific Publishers, Oxford, UK. pp. 125-135

Turner, N. C., and M. M. Jones. 1980. Turgor maintenance by osmotic adjustment. In: Adaptations of plants to water and high temperature stress. Turner NC, Kramer PG (eds). John Wiley, New York., pp. 87-103.

Valentovic, P., M. Luxova, L. Kolarovic and O. Gasparikova. 2006. Effect of osmotic stress on compatible solutes content, membrane stability and water relations in two maize cultivars. Plant Soil Environ. 4: 186-191.

Vendruscolo, A.C. G., I. Schuster, M. Pileggi, C. A. Scapim, H.B.C. Molinar, C. J. Marur and L. G. C. Vieira. 2007. Stress-induced synthesis of praline confers tolerance to water deficit in transgenic wheat. J. Plant. Physiol. 164(10): 1367-1376.

Wang, Y., J. Ying, M. Kuzma, M. Chalifoux, A. Sample, C. McArthur, T. Uchacz, C. Sarvas, J. Wan, D. T. Dennis, P. McCourt and Y. Huang. (2005). Molecular tailoring of farnesylation for plant drought tolerance and yield protection. Plant J. 43: 413-24.

Williamson, J. D., and R.S. Quatrano. 1988. ABA regulation of two classes of embryo specific sequences in mature wheat embryos. Plant Physiol. 86: 208-215.

Zhang, H., M. Q. Qiu, G. X Wang, Y. Gan, K. F. Zheng and X. G. Yao. 2009. The effect of sensitivity to absicic acid on stomatal behavior in Arabidopsis thaliana. EurAsian J. BioSci. 3: 10-16.

Zhang, J., W. Jia, J. Yang and A. M. Ismail. (2006). Role of ABA in integrating plant responses to drought and salt stress. Field Crop Res. 97: 111-119. 